\title{
EXPERIMENTAL INVESTIGATION OF GENERIC ICE BLOCK TRAJECTORIES UNDER FREESTREAM CONDITIONS
}

\author{
T. Kilian \\ DLR (German Aerospace Center), \\ Institute of Aerodynamics and Flow Technology, \\ Lilienthalplatz 7, 38108 Braunschweig, Germany
}

\begin{abstract}
An experimental database of trajectories featuring idealized, simplified artificial ice blocks under freestream conditions has been created. It includes a variation of key parameters like the ice block geometry, density, freestream velocity and release angle of attack. The ice block's position and attitude were optically tracked using a system of two high-speed cameras and a dedicated post-processing routine. The work performed in the scope of the EU co-funded research project STORM aims at the validation and further development of current simulation tools for ice release and ice block trajectories in terms of their ability to cope with the high complexity and randomness of this phenomenon. These tools of different level of fidelity are currently used during the aircraft certification process to estimate the thread of large ice pieces released from the aircraft's surface impacting into downstream components. The major experimental findings are discussed in this article alongside first promising results from numerical trajectory simulations.
\end{abstract}

Keywords: Aircraft Icing, Ice Block Trajectories, Experimental Aerodynamics

\section{NOMENCLATURE}

CFD Computational Fluid Dynamics

DLR German Aerospace Center

DNW German-Dutch Wind Tunnels

IPS Ice Protection System

NWB Low-speed wind tunnel Braunschweig

$q$

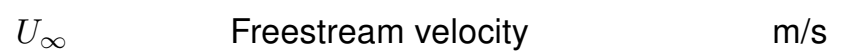

$X, Y, Z \quad$ Axis coordinates $\quad \mathrm{m}$

$\alpha_{R} \quad$ Release angle of attack deg

$\Delta \mathrm{t} \quad$ Physical time step size

$\rho_{\text {Ice }} \quad$ Ice block density

$\Theta$
Rotational velocity around $\mathrm{Y}$-axis deg/s

s

$\mathrm{g} / \mathrm{cm}^{3}$

Pitch angle

\section{INTRODUCTION}

Ice release is of concern to aircraft manufacturers due to the potential damage that the ice debris can cause on aircraft components. Impact of large ice debris particles on downstream aerodynamic surfaces and ingestion into aft mounted engines must be considered during the aircraft certification process. Those particles typically result from ice accumulation on unprotected surfaces, ice accretions downstream of ice protected areas or ice growth on surfaces due to delayed activation or failure of ice protection systems (IPS). This raises the need for accurate ice trajectory simulation tools to support pre-design, design and certification phases while improving cost efficiency.

There are currently two types of models used to track shed ice pieces, which are distinguished by their level of representation of fluid - body interaction. Basic application schemes of both types are depicted in Fig. 1.

The first type of models is characterized by a oneway approach, neglecting the ice shape's interference with the surrounding flow field, therefore herein referred to as "low-fidelity". These models, as described deg in [1] make use of an approach, in which the forces and 


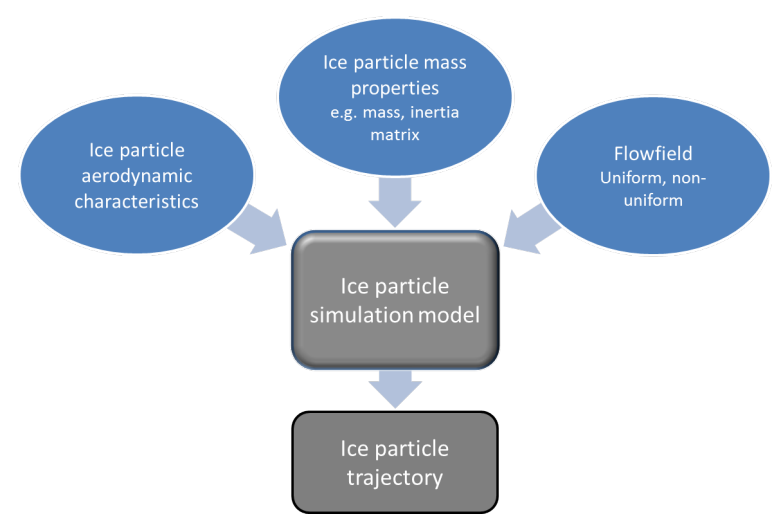

a) Low-fidelity method: One-way approach without iterative coupling.

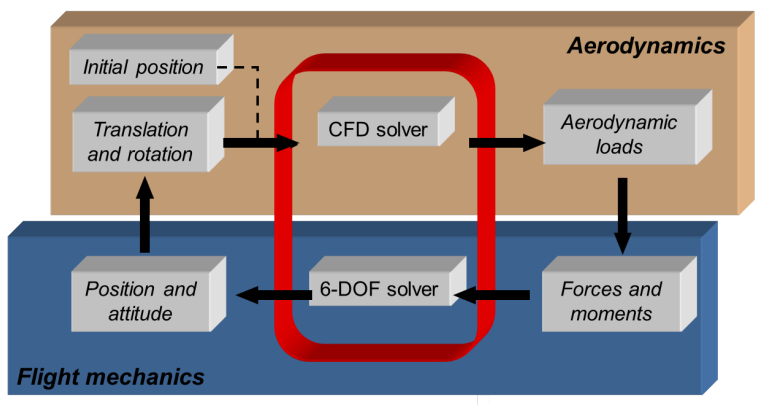

b) High-fidelity method: Aerodynamics and flight mechanics fully coupled.

Fig. 1: Basic application schemes of ice block trajectory simulation methods.

moments acting on the particle are extracted locally from the steady flow field, typically pre-computed by CFD methods. As mentioned above, the particle itself is not expected to significantly affect the surrounding airflow, which makes an expensive iterative recomputation routine unnecessary. The aerodynamic and mass properties of the ice shape are typically evaluated experimentally or analytically. The trajectory is computed by integrating the translational and rotational movements due to forces and moments acting on the ice shape. Major advantages of low-fidelity models lie in the low computational costs, which enables the use of statistical approaches like the Monte-Carlo method to capture the randomness of the process. They are currently state-of-the-art within the aircraft certification process, although inherently lacking the exact representation of complex flow topologies.

The so-called "high-fidelity" models are based on an strong coupling cycle consisting of both, aerodynamics and rigid body dynamics solvers, resulting in a more physical representation of the fluid - body interaction. The time accurate, two-way interaction between the ice particle and the surrounding flow is computed requiring the use of emerging simulation tools, such as approaches based on immersed boundary methods [2], penalization and level sets [3] or chimera grids [4]. The flow field and the ice debris movements are computed iteratively in a coupled manner using basic mechanical laws, no database interpolation is needed, as it is the case for low-fidelity methods. While being potentially more accurate than low-fidelity methods in a complex and unsteady flow environment, the computational effort is much larger, making parameter variations costly.

Present ice trajectory simulation tools of low and high fidelity have limited capabilities due to the lack of appropriate aerodynamic force and moment data for ice fragments and the large number of variables that can affect the trajectories of ice particles like shape, size, mass, initial velocity and shedding location. Furthermore, both methods rely heavily on experimental trajectory data for validation purposes. Although there has been a large number of ice block trajectory tests being carried out by Papadakis et. al. as described in [1], [5] and [6], there is no public database available. In order to generate a trajectory database of generic but realistic ice blocks under freestream conditions a wind tunnel test was performed by the German Aerospace Center (DLR) within the scope of the project STORM [7]. STORM (efficient ice protection Systems and simulation Techniques Of ice Release on propulsive systeMs) is a 3-year collaborative research project comprising 14 research and industrial partners from 7 European countries.

\section{EXPERIMENTAL SETUP}



Fig. 2: Sketch of the test setup inside the closed test section of the DNW-NWB.

The trajectory study was conducted at the low-speed aero-acoustics wind tunnel facility NWB (Low-speed wind tunnel Braunschweig), which is operated by the foundation DNW (German-Dutch Wind Tunnels) at 
the DLR site in Braunschweig, Germany. The NWB is an atmospheric wind tunnel with a closed return circuit and can be operated optionally with a closed, slotted or open test section at a maximum speed of $90 \mathrm{~m} / \mathrm{s}$. The DNW-NWB was chosen because of its testing capabilities and DLR's and NWB's experience in performing such tests (see [8] and [9]).

This particular test was performed within the closed test section with a height of $2.8 \mathrm{~m}$ and a width of $3.25 \mathrm{~m}$. The artificial ice block was released at the center-line of the closed test section $400 \mathrm{~mm}$ from the ceiling in order to stay clear of the boundary layer, which is approximately $90 \mathrm{~mm}$ thick at that point and at the same time have a maximum falling distance of 2.4 meters.

The test section was equipped with sponge layers on the bottom (thickness: $70 \mathrm{~mm}$ ) and ceiling (thickness: $50 \mathrm{~mm}$ ) featuring a $30^{\circ}$ slope at the front and a safety net approximately 3 meters behind the release point preventing the ice blocks from impacting into the downstream wind tunnel structure. A sketch of this setup is shown in Fig. 2.

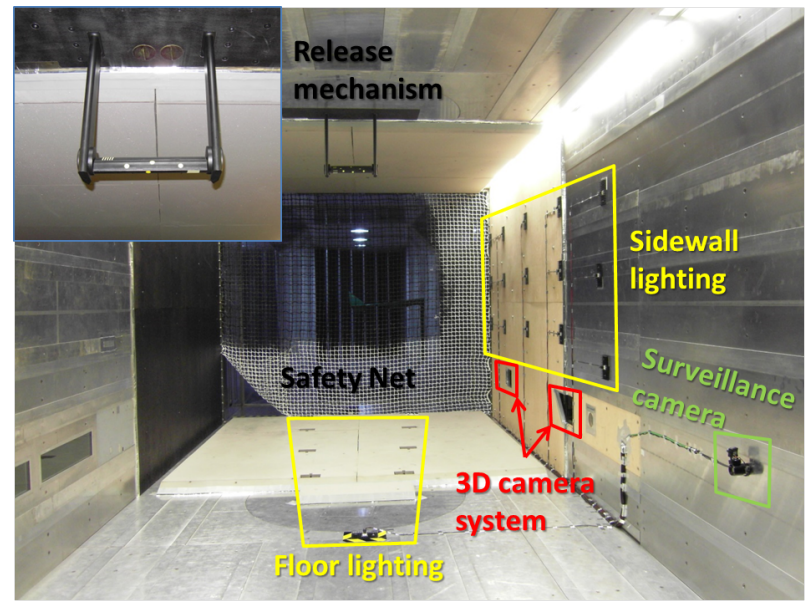

Fig. 3: Streamwise view of the test section setup.

Two high-speed cameras for trajectory tracking are placed behind glass windows at the right side wall. More details on the camera and data acquisition system are given in section 3 . The use of high-speed cameras made a heavy lighting of the trajectory region necessary. 22 high-energy UV-LEDs of 30 Watts each were distributed on the floor and side wall (see Fig. 3). The UV lighting in combination with special fluorescent markers and camera filters made it possible to reduce misinterpretations due to unwanted background reflections to a minimum.

\subsection{RELEASE MECHANISM}

A test mount has been custom-made in order to assure the tight and vibration-free fixation of the ice block with the possibility to set any desired angle of attack. A major design requirement was the avoidance of additional forces or moments acting on the ice block at the moment of release, since the test results are potentially influenced by those initial conditions.

The design features two aerodynamically shaped struts extruding from the ceiling for minimum flow disturbance. Rotary brackets are attached on either side to set the angle of attack. The ice block is held in place by four spring-loaded and pneumatically driven bolts to ensure tight fixation and symmetric release. An overview of the design including a detailed view of the rotary bracket and fixation bolts is shown in Fig. 4 .
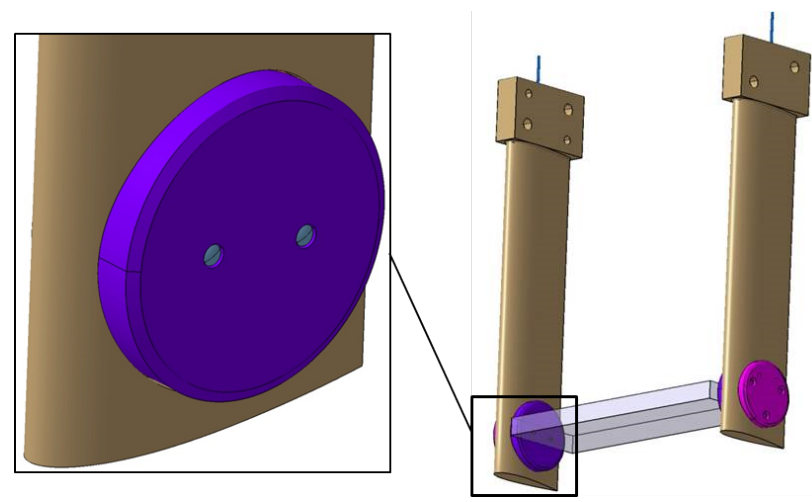

Fig. 4: Design of the release mechanism.

\subsection{ICE SHAPE SELECTION AND MANUFACTUR- ING}

Two ice geometries, which are representative for wing leading edge icing under a wide range of accretion conditions, were chosen for trajectory testing. Their 2D-shape is depicted in Fig. 5 also defining the $\alpha=0^{\circ}$ orientation.

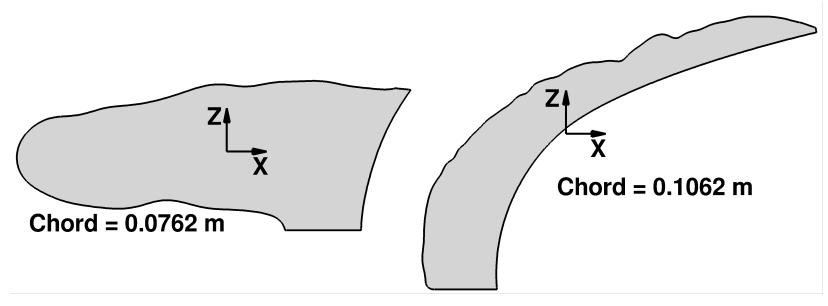
a) 1-Horn
b) GLC305-rime

Fig. 5: Overview of tested ice shapes.

The 1-Horn shape represents the upper horn of a 3 -inch glace ice shape on a wing's leading edge. It is the Nlf 0414 airfoil - case 8030636 from the validation database of the ice accretion code LEWICE [10], where the horn has been scaled to 3 inches. The second shape represents a rime ice layer, which can also be found on the upper part of a wings leading edge, but under different humidity and temperature conditions. It is taken from the same database, case 073101 featuring a GLC 305 airfoil. Details concerning the ice accretion conditions can be found in [10]. 


\begin{tabular}{|l|c|c|c|c|c|c|c|c|c|}
\hline Ice Shape & Density & Weight & \multicolumn{1}{|c|}{ Ixx } & Iyy & Izz & Ixy & \multicolumn{2}{|c|}{ Ixz } & Iyz \\
\cline { 5 - 9 } & {$\left[\mathrm{g} / \mathrm{cm}^{3}\right]$} & {$[\mathrm{gg}]$} & \multicolumn{5}{|c|}{$\left[\mathrm{kg} \mathrm{m}^{2}\right]$} \\
\hline 1-Horn light & 0.8513 & 454.5 & $3.0 \mathrm{E}-03$ & $2.248 \mathrm{E}-04$ & $3.0 \mathrm{E}-03$ & 0 & $-6.002 \mathrm{E}-06$ & 0 \\
\hline 1-Horn heavy & 0.9204 & 491.4 & $3.0 \mathrm{E}-03$ & $2.317 \mathrm{E}-04$ & $4.0 \mathrm{E}-03$ & 0 & $-6.173 \mathrm{E}-06$ & 0 \\
\hline GLC305-rime light & 0.8747 & 407.5 & $3.0 \mathrm{E}-03$ & $4.259 \mathrm{E}-04$ & $3.0 \mathrm{E}-03$ & 0 & $-1.782 \mathrm{E}-06$ & 0 \\
\hline GLC305-rime heavy & 0.9438 & 440.7 & $3.0 \mathrm{E}-03$ & $4.533 \mathrm{E}-04$ & $3.0 \mathrm{E}-03$ & 0 & $-1.911 \mathrm{E}-06$ & 0 \\
\hline
\end{tabular}

Tab. 1: Measured mass and inertia properties of the ice blocks including cavities, side-holes and paint finish.

The ice shape sizing corresponds to the typical ice thicknesses accreted on an aircraft surface. Due to wind tunnel restrictions the length is limited to $300 \mathrm{~mm}$. In order to investigate the influence of ice density variations on the trajectories, there was a set of bodies made at the upper ("heavy": $\rho_{I c e}=0.92 \mathrm{~g} / \mathrm{cm}^{3}$ ) and one at the lower ("light": $\rho_{\text {Ice }}=0.85 \mathrm{~g} / \mathrm{cm}^{3}$ ) limit of the range of typical ice densities. The change in density was realized by the introduction of cavities within the ice shape symmetrical to the CG-position during the rapid prototyping process. Due to those cavities some parts of the inertia matrix are slightly altered in comparison to an ice block with uniform mass distribution. The ice shapes were spray-painted black and finished using steel wool in order achieve a smooth and yet non-reflective surface. See Tab. 1 for the final mass properties including all internal cavities, side holes for model fixation and paint finish.

\section{DATA ACQUISITION AND POST-PROCESSING}

A system of two Mikrotron EoSens CoaXPress 4CPX6 cameras equipped with $12 \mathrm{~mm}$ lenses was used to capture the trajectories and attitudes of the ice blocks. They deliver black/white images at a resolution of 4 Megapixels (2336 $\times 1728$ pixels) at a frame rate of $500 \mathrm{fps}$. The image sets were processed and stored in real-time using a Silicon Software CoaXPress frame grabber. One camera was placed slightly upstream of the release point and the second one 3 meter downstream towards the rear of the measurement volume $50 \mathrm{~cm}$ above the tunnel floor. This setup results in a $3 \mathrm{D}$-angle of nearly $60^{\circ}$, which is favorable for accurate position measurements in every direction.

The translational and rotational movements are tracked by observing a set of circular fluorescent markers attached to every side of the ice shape's surface. Additional stripe patterns were added as a backup solution for automated attitude evaluation, yet not needed during this test. Fig. 6 shows both ice shapes ready for testing.

The specialized software package picCOLOR, developed by F.I.B.U.S. [11], allows for the determination of the 3D position of every marker placed on the moving body by computationally evaluating two corresponding marker images. For this to be possible a set of at least 3 markers have to be clearly visible from either camera. Additional geometrical requirements

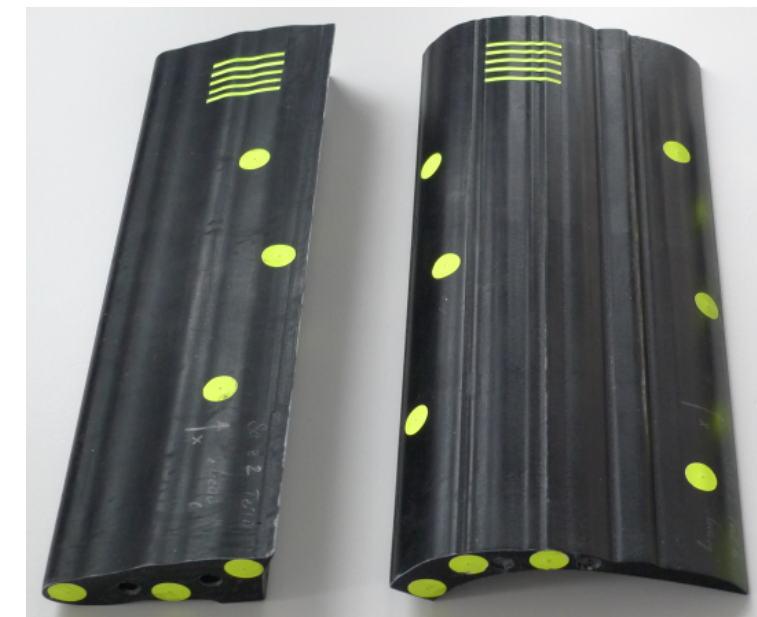

Fig. 6: Ice shape 1-Horn (left) and GLC305-rime (right) ready for testing.

related to a special routine for marker identifications make the data acquisition in the case of rotating bodies not trivial. Since the markers were small compared to the large area of observation and rarely in an ideal position for observation, special attendance had to be put on the settings for image post-processing. An exemplary sequence of raw images (black/white inverted) in Fig. 7 illustrates the typical visibility conditions during the tests. picCOLOR has already been successfully applied to trajectory testing at $D L R$ in the past (see [12]).

Camera 1 (upstream)

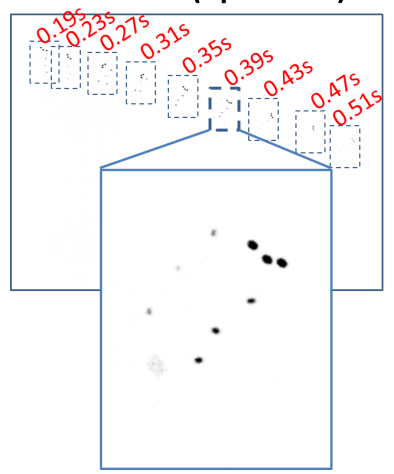

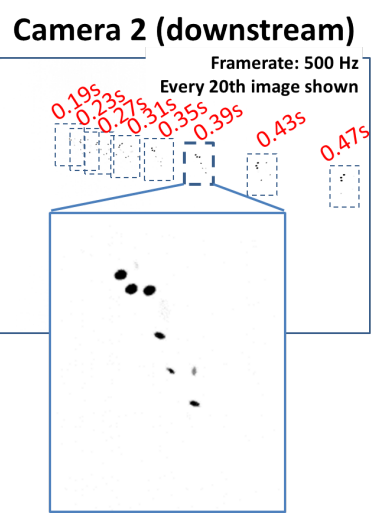

Fig. 7: Exemplary sequence of raw image sets for trajectory analysis.

A subsequent post-processing routine has been developed, which processes the raw marker position data into time-resolved information about the center of gravity as well as the attitude of the moving ice block [13]. 


\section{EXPERIMENTAL RESULTS}

The ice shapes were released with a variation of release angle of attack $\left(0^{\circ}, \pm 30^{\circ}\right)$, density $(0.85$, $\left.0.92 \mathrm{~g} / \mathrm{cm}^{3}\right)$ and freestream velocity $(20,40,50 \mathrm{~m} / \mathrm{s})$. The measurement time was 1 second at a frame rate of $500 \mathrm{~Hz}$. The ice block was automatically released within 0.2 seconds after the measurement started. The traveling time through the test section ranged in the order of 0.25 to 0.5 seconds ( $\approx 125-250$ image-sets) mainly depending on the freestream velocity.

The reader will notice some occasional peaks within the shown graphs especially when looking at the pitch data. Those are not physical movements of the ice shape, but rather difficulties of the post-processing software in finding the correct orientation of the geometry due to an uncertain identification of surface markers.

\subsection{VARIATION OF MAIN TEST PARAMETERS}

In the following section the influence of the four main test parameters on the trajectories is discussed. Since the flow conditions as well as the ice shapes are symmetrical to the XZ-plane parallel to the wind-tunnel side walls, the investigations are mainly focused on the XZ-position of the body's center of gravity and its pitch angle $\Theta$ over the streamwise coordinate $X$.

\subsubsection{Ice block geometry}

From unpublished results of a dedicated wind-tunnel test performed within the scope of the project STORM the aerodynamic characteristics of both ice shapes are known to be clearly different. This is bound to have a major effect on their trajectories.

The trajectories of the heavy version of both ice shapes at a release angle of attack of $\alpha_{R}=0^{\circ}$ and a freestream velocity of $U_{\infty}=40 \mathrm{~m} / \mathrm{s}$ are compared in Fig. A.1. The trajectory slope of both shapes is nearly linear. After rapidly pitching down after release the GLC305-rime shape starts performing a damped oscillation around a mean pitch angle of $-45^{\circ}$, where static tests have revealed a change of sign in pitching momentum. Drag forces peak at this pitch angle making the trajectory slope angle relatively small. The 1-Horn shape continues a fast negative pitching motion performing over 5 full $360^{\circ}$ revolutions within 2.5 meters of travel, which corresponds to a maximum pitch rate of $q=10,000$ degrees/s. The overall lower drag of the 1-Horn shape alongside the pitch-induced Magnus effect creating an additional force in downward direction make the slope considerably steeper. At a downstream position of 2 meters the lateral distance of both ice shapes is 0.75 meters, which corresponds to a difference in slope angle of $17^{\circ}$.

\subsubsection{Freestream velocity}

Trajectories of bluff bodies are mainly influenced by gravitational and drag forces. The latter is influenced by the freestream velocity through a quadratic relation $\operatorname{Drag}=f\left(U_{\infty}^{2}\right)$. The dominant influence of the freestream velocity on the trajectories of both iceshapes at $\alpha_{R}=0^{\circ}$ can clearly be observed in Fig. A.2.

The geometrical trends described in section 4.1.1 hold for the range of tested velocities. The slope angle of the trajectory decreases significantly when going from $U_{\infty}=20$ to $40 \mathrm{~m} / \mathrm{s}$. The decrease is much smaller when proceeding to $50 \mathrm{~m} / \mathrm{s}$. With the gravitational component remaining constant the slope angle is expected to converge towards $0^{\circ}$ (horizontal) at higher velocities, e.g. realistic inflight conditions. Pitching velocities are aerodynamically induced and thus increase with the freestream velocity, which results in a higher pitching frequency for the GLC305-rime shape and an overall higher negative pitch rate for the 1-Horn shape.

\subsubsection{Release angle of attack}

The forces and moments acting on the ice shape before release are highly dependent of the angle of attack at which it is attached to the release mechanism. Those forces and moments in combination with gravitational forces determine the body's initial acceleration at the moment of release.

The influence of the release angle of attack at a freestream velocity of $40 \mathrm{~m} / \mathrm{s}$ on the trajectory of the GLC305-rime shape is highlighted in the left part of Fig. A.3. The trajectory path moves further up with increasing release angle of attack. From static aerodynamic characteristics tests can be derived that this is due to a strong increase of forces in positive $X$ and Z-direction (drag and lift). The amplitude of the pitch oscillation decreases when going from $\alpha_{R}=+30^{\circ}$ to $-30^{\circ}$, which is closer to the uninfluenced median pitch angle of $-45^{\circ}$.

The right part of Fig. A.3 shows the 1-Horn shape's trajectory being more heavily influenced by the release angle of attack. The slope angle for $\alpha_{R}=-30^{\circ}$ and $30^{\circ}$ is considerably smaller than for $0^{\circ}$. At $x=2 \mathrm{~m}$ there is an offset of 1 meter between the trajectories, which corresponds to a slope increase of $25^{\circ}$. There is also a large influence on the pitching motion. While the $0^{\circ}$ case performs a constant negative rotation the ice shape changes the rotational direction several times at $\alpha_{R}=-30^{\circ}$ and $30^{\circ}$. For both ice shapes, the same trends can be observed for different velocities of 20 and $50 \mathrm{~m} / \mathrm{s}$.

\subsubsection{Ice shape density}

The heavy and light versions of the ice shapes cover the range of natural ice densities. The relative change in mass is $8 \%$. The influence of this change in density 
on the trajectory and pitch angle of the GLC305-rime shape is small as depicted in Fig. A.4. There is a slight reduction $\left(<1^{\circ}\right)$ in the trajectory slope angle at 40 and $50 \mathrm{~m} / \mathrm{s}$ for the light version. At $20 \mathrm{~m} / \mathrm{s}$ the difference in slope angle rises to $2^{\circ}$ as mass inertia related effects become more dominant. The pitch rates are slightly higher for the low density ice blocks, which results in a lower pitching frequency for the GLC305-rime shape and an overall lower pitch down angle for the 1Horn shape (see Fig. A.5). This effect again becomes more dominant at lower freestream velocities. It can be stated, that the variation of ice density within the boundaries of natural ice only has a small effect on the trajectory at low speeds and becomes more and more negligible as the speed increases, e.g. towards realistic inflight conditions.

\subsection{DATABASE QUALITY}

The ability to validate numerical tools against an experimental database is highly dependent on its quality in terms of resolution, accuracy and repeatability.

\subsubsection{Temporal resolution}

The trajectories were tracked with a frame rate of $500 \mathrm{~Hz}$. The maximum measured ice block velocity in the rear part of the test section was at $19 \mathrm{~m} / \mathrm{s}$, which corresponds to an ice block movement of $38 \mathrm{~mm}$ between two images, less than half of a chord length. The maximum rotational velocities at $10,000 \mathrm{deg} / \mathrm{s}$ were achieved by the 1-Horn shape, pitching 22 degrees between two frames. This can be considered a high enough resolution to capture the main trajectory and orientation parameters.

\subsubsection{Measurement accuracy}

The nominal accuracy of the optical measurement routine is at $1 / 10$ of a pixel, which corresponds to $0.1 \mathrm{~mm}$ given the camera resolution and the mean observation distance. Yet, this nominal value may be degraded due to non-optimal lighting and visibility conditions.

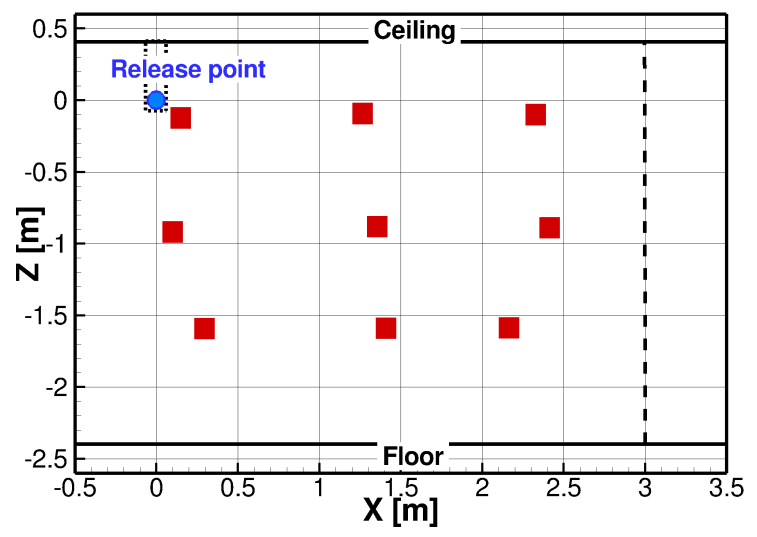

Fig. 8: Marker positions for accuracy tests.
In order to gain a more realistic idea of the measurement accuracy under the given setup conditions a dedicated test was performed. A set of markers as used during the trajectory tests was placed at various fixed positions across the center-line of the trajectory region (see Fig. 8). The maximum deviation of position data during a 500 image sequence gives a good indication of the local measurement accuracy. The spread of the measured CG-positions in the $X Y$ and $X Z$ plane is depicted in Fig. A.6. The measurement accuracy decreases linearly with increasing distance to the cameras, which are placed in the lower part of the side wall. Therefore the upper test points have got a higher root mean square deviation in the $Y$ and $Z$ direction of $0.2 \mathrm{~mm}$ compared to the lower test points, which are close to the nominal value of $0.1 \mathrm{~mm}$. The camera positioning relative to the test section makes the deviation in $\mathrm{X}$ direction being less affected by the measurement distance, remaining constant at $0.1 \mathrm{~mm}$. Thus, the actual relative accuracy of the tracking system is within $0.1-0.2 \%$ of the ice block's reference length. When taking into account a marker distance ranging at $50-100 \mathrm{~mm}$ for attitude evaluation through triangulation the maximum angular deviation range is at $0.25-0.5^{\circ}$.

When observing rapidly moving particles, motion blur may impair the accuracy of image processing. In order to minimize this potential thread, a high shutter speed of $0.5 \mathrm{~ms}$ was chosen. This setting results in a motion blur at a maximum observed ice block traveling speed of $19 \mathrm{~m} / \mathrm{s}$ of less than a marker diameter. Those slightly stretched marker images can be handled by picCOLOR without accuracy reduction.

\subsubsection{Repeatability}

Every trajectory test was repeated several times in order to gain information about the level of repeatability. The trajectory graphs in section 4.1 are a superposition of 7 to 10 subsequent tests. From visual inspection the overall level of repeatability can be considered as very high.

For both ice shapes at a release angle of attack of $\alpha_{R}=0^{\circ}$ and a freestream velocity of $V=40 \mathrm{~m} / \mathrm{s}$ the repeatability can be assessed by calculating the maximum root mean square (RMS) deviation of position and attitude data at a rear location of the test section. The results are summarized in Tab 2. For the GLC305-rime shape the lateral deviation is at $4 \mathrm{~mm}$ and the pitch deviation at $2.5^{\circ}$. Due to the more dynamic movement of the 1-Horn shape the deviation value are higher at $17 \mathrm{~mm}$ and $13^{\circ}$. But when ignoring one single off lying curve, which can be explained by issues with symmetrical release in the early test phase, the lateral deviation comes down to $3 \mathrm{~mm}$ (GLC305rime) and $7 \mathrm{~mm}$ (1-Horn). Thus, the maximum relative deviation in the rear part of the test section is in the 
order of $3-9 \%$ of the ice block's reference length.

\begin{tabular}{|l|c|c|}
\hline Ice Shape & \multicolumn{2}{|c|}{ RMS deviation at $X=2 \mathrm{~m}$} \\
& $Z[\mathrm{~mm}]$ & Pitch [deg] \\
\hline GLC305-rime & $3(4)$ & 2.5 \\
\hline 1-Horn & $7(17)$ & 13 \\
\hline
\end{tabular}

Tab. 2: RMS values of lateral and pitch deviation at $\mathrm{X}=2 \mathrm{~m}\left(\mathrm{~V}=40 \mathrm{~m} / \mathrm{s} ; \alpha_{R}=0^{\circ}\right)$

With that it can be stated, that the temporal resolution is sufficient and the overall level of accuracy and repeatability is very high, which makes the database valuable for the validation of numerical tools.

\section{NUMERICAL TRAJECTORY SIMULATION}

Unsteady URANS-based trajectory simulations of the ice shapes under freestream conditions are currently ongoing. First very promising results are described below.

\subsection{NUMERICAL SETUP}

The 3D computations of the ice block trajectories were carried out with the Reynolds-averaged Navier-Stokes (RANS) solver TAU [14], developed by DLR using unstructured hybrid meshes. It was coupled to an integrated six degrees of freedom rigid body dynamics (RBD) module. A so called "strong coupling" approach was applied, which means that the coupled equations are iteratively solved within every physical time step by repeatedly solving the involved disciplines CFD and RBD separately. This coupling routine has been validated and successfully applied to several generic test cases as well as store release and gust encounter scenarios as described in [12], [15], [16] and [17].

Due to the largely detached and oscillating flow conditions in the ice shape's wake, unsteady computations were necessary using the dual-time stepping approach. Fig. 9 gives an impression of the complexity of the flow field around the GLC305-rime shape. Extensive numerical studies have proven a physical time step of $\Delta t=0.0001$ seconds to be necessary to correctly resolve the unsteadiness of the flow. This corresponds to $1 / 20^{\text {th }}$ of the experimental frame rate. The maximum number of inner iterations was limited to $100-150$ by the Cauchy convergence criterion, monitoring the fluctuations in the integral values of lift, drag and pitching moment coefficient. The numerical studies also involved the influence of turbulence modeling. The one-equation Spalart-Allmaras model [18] in the negative formulation (SA-neg), Menter's two-equation Shear-Stress-Transport model [19] (kw-SST) as well as the Launder-Reece-Rodi-Type Reynolds-Stress turbulence model [20] (RSM-LRR) using the g-formulation were tested. The RSM-LRR model turned out to be most suited for this specific application in terms of stability and result quality and it was therefore used for all trajectory simulations. The geometry of the release mechanism is neglected because of its aerodynamic shape and since its wake position is not expected to largely interfere with the trajectory path.

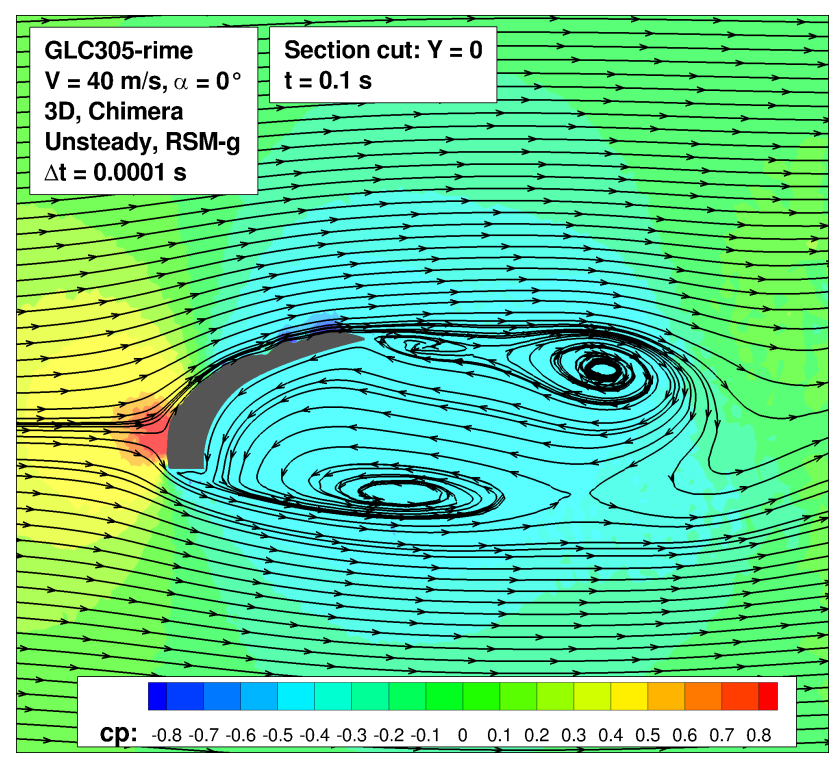

Fig. 9: Pressure distribution and streamlines in the vicinity of the GLC305-rime shape.

Hybrid numerical meshes consisting of structured, unstructured and semi-structured cells were generated with the commercial mesh generator CENTAUR by CentaurSoft [21]. The chimera technique featuring a semi-automatic hole cutting algorithm based on hole definition geometries was applied to simulate the ice shape's movement relative to a background mesh. The background mesh including refinements within the trajectory region and the integrated ice block mesh comprises 3.54 million nodes.

\subsection{COMPARISON TO EXPERIMENTAL RE- SULTS}

The heavy version of the GLC305-rime shape at a freestream velocity of $U_{\infty}=40 \mathrm{~m} / \mathrm{s}$ and a release angle of attack of $\alpha_{R}=0^{\circ}$ was chosen for the initial computations, because of its known complex pitching motion and it being the potentially more challenging geometry to simulate. The initial 0.5 seconds after release were simulated, which relates to 5000 physical time-steps. The computational run-time was 122 hours on 288 CPUs.

A comparison of numerical and experimental results as a function of the streamwise coordinate $X$ is given in Fig. 10. Over the complete measurement region the numerical results are in very good agreement to the experimental curves. The pitching amplitude is slightly over-predicted by $4^{\circ}$, yet without any visible effect on the $X Z$ trajectory, which lies exactly within the spread of the experimental curves. 


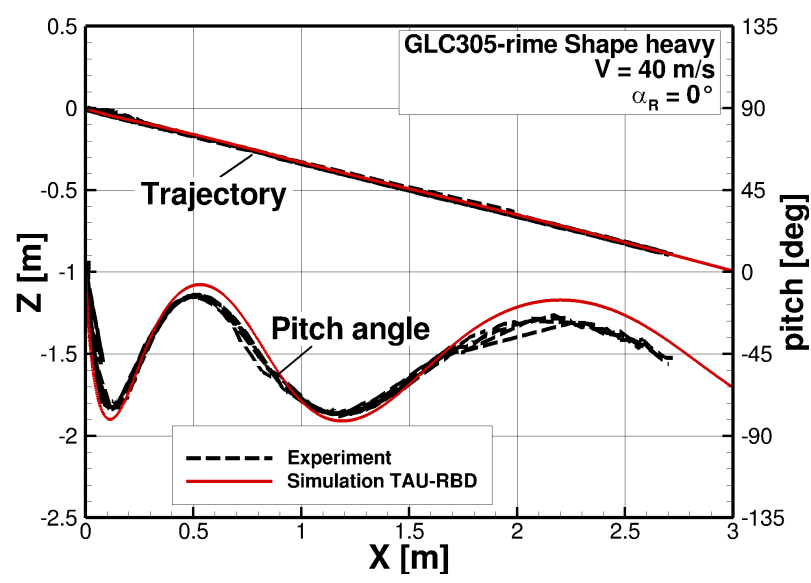

Fig. 10: Comparison of experimental and numerical results for trajectory and pitch angle.

\section{CONCLUSION}

A trajectory test campaign featuring idealized, simplified artificial ice blocks has been performed at the low-speed wind tunnel facility DNW-NWB in Braunschweig/Germany. The trajectories of two ice shapes were optically tracked, the upper part of a 3-inch hornice shape as well as a shape resembling a thin rime-ice layer. In addition to the shape variation the influence of three parameters was analyzed: release angle of attack, freestream velocity and ice block density. Three of these parameters have a distinct effect on the ice block trajectory and pitching motion. The shape of the ice block is the most dominant parameter having a large effect throughout the tested velocity range. The change in ice density of $8 \%$, which represents the natural spread of ice densities, has shown to be too small to clearly influence the trajectory especially at higher speeds and may therefore be neglected in aircraft applications.

From dedicated test on measurement accuracy a spatial deviation throughout the test section of less than $0.2 \%$ of the ice block's reference length was derived, which corresponds to a rotational deviation of less than $0.5^{\circ}$. The trajectory spread of up to 10 repeatability tests per setup was below 3 to $9 \%$ of the ice blocks reference length. The observed quality of the data as well as the level of repeatability can therefore be considered as very high and well suited for numerical tool validation.

First high-fidelity ice block trajectory simulations using the DLR TAU Code coupled to an imbedded RBD module were successful. The computed movements of the GLC305-rime shape are within the spread of the experimental data. Thus, it has been proved, that the applied numerical process is applicable for the computation of complex ice block trajectories. Further numerical investigations using methods of different levels of fidelity are currently ongoing within the scope of the STORM project.

\section{ACKNOWLEDGMENTS}

The work presented in this paper was carried out in the scope of the EU project STORM and has received funding from the European Union's Seventh Framework Programme FP7 2007-2013 under grant agreement no. 605180 .

The author like to express his gratitude to the entire team of the DNW-NWB wind tunnel facility in Braunschweig alongside Mr. Müller from F.I.B.U.S. for their very strong support during the test campaigns. Furthermore, special thanks go to Björn Gräfer for his valuable effort in processing the trajectory data.

\section{REFERENCES}

[1] Papadakis, M., Yeong, H.W., Suares, I.G. and Jacob, J.: "Experimental and Computational Investigation of Ice Shedding from Aircraft Surfaces". AIAA Paper 2006-1010, 44th AIAA Aerospace Science Meeting and Exhibit, Reno, Nevada, 9 12 January 2006.

[2] Mittal, R. and laccarino, G.: "Immersed Boundary Methods". Annual Review of Fluid Mechanics, vol. 37, issue 1, pp. 239-261, 2005.

[3] Morency, F., Beaugendre, H., Gallizio, F. and Laurens, S.: "Computation of Ice Shedding Trajectories Using Cartesian Grids, Penalization, and Level Sets". Modelling and Simulation in Engineering, Volume 2011, Article ID 274947, 2011.

[4] Madrane, A., Stürmer, A. and Raichle, A.: "Parallel Implementation of a Dynamic Overset Unstructured Grid Approach". In: Proc. of "European Conf. on Computational Fluid Dynamics", ECCOMAS CFD, Jyväskylä, Finland, July 24-28 2004.

[5] Papadakis, M., Yeong, H. and Suares, I.G.: "Simulation of Ice Shedding from a Business Jet Aircraft". AIAA Paper 2007-506, 45th AIAA Aerospace Science Meeting and Exhibit, Reno, Nevada, 8 - 11 January 2007.

[6] Papadakis, M., Yeong, H. and Shimoi, K.: "Ice Shedding Experiments with Simulated Ice Shapes". AIAA Paper 2009-3972, 1st AIAA Atmospheric and Space Environments Conference, San Antonio, Texas, 22 - 25 June 2009.

[7] STORM Consortium Website: www.fp7-storm.eu, retrieved: 01.08.2016.

[8] Loeser, T. and Bergmann, A.: "Capabilities of Deployment Tests at DNW-NWB". RTO Meeting Proceedings RTO-MP-AVT-133, 2006. 
[9] Geisbauer, S., Schade, N., Enk, S., Schmidt, H. and Arnold, J.: "Experimental and Numerical Research of the Aerodynamics of Unsteady Moving Aircraft". AIAA Paper 2011-2565, 21st AIAA Aerodynamic Decelerator Systems Technology Conference and Seminar, Dublin, Ireland, May 2011.

[10] Wright, W. B. and Rutkowski, A.: "Validation Results for LEWICE 2.0”. NASA Contractor Report (NASA/CR) 1999-208690, 1999.

[11] F.I.B.U.S. Website: www.fibus.org, retrieved: 01.08.2016.

[12] Schade, N.: "Simulation of Trajectories of Cuboid Cargos Released from a Generic Transport Aircraft”. AIAA Paper 2011-3959, 29th AIAA Applied Aerodynamics Conference, Honolulu, Hawaii, $27-$ 30 June 2011.

[13] Gräfer, B.: "Experimentelle Untersuchung von Trajektorien generischer Eiskörper in ungestörter Anströmung". Bachelor Thesis, University of Braunschweig, 2015.

[14] Schwamborn, D., Gerhold, T. and Heinrich, R.: "The DLR TAU-Code: Recent Applications in Research and Industry". In: Proc. of "European Conf. on Computational Fluid Dynamics", ECCOMAS CFD, Delft, The Netherlands, 2006.

[15] Heinrich, R. and Michler, A.: "Unsteady simulation of the encounter of a transport aircraft with a generic gust by CFD flight mechanics coupling". In: Proc. of CEAS 2009 European Air and Space Conference, Manchester, UK, October 26292009.

[16] Reimer, L., Heinrich, R. and Meuer, R.:"Validation of a Time-Domain TAU-Flight Dynamics Coupling based on Store Release Scenarios". Notes on Numerical Fluid Mechanics and Multidisciplinary Design, vol. 124, pp. 455-463, 2014.

[17] Geisbauer, S., Bier, N., Kirz, J. and Roosenboom, E. W. M.: "Validation of the Flow Topology Around Several Airdrop Cargo Configurations at Static Conditions". AIAA Paper 2013-3155, 31st AIAA Applied Aerodynamics Conference, San Diego, CA, June 24-27 2013.

[18] Spalart, P. R. and Allmaras, S. R.: "A oneequation turbulence model for aerodynamic flows". La Recherche Aerospatiale, vol. 1, issue 1, pp. 5-21,1994.

[19] Menter, F. R.: "Zonal Two Equation k-w Turbulence Models for Aerodynamic Flows". AIAA Journal, vol. 32, no. 8, pp. 1598-1605, 1994.
[20] Launder, B. E., Reece, G. J. and Rodi, W.: "Progress in the development of a Reynoldsstress turbulence closure". Journal of Fluid Mechanics, vol. 63, pp. 537-566, 1975.

[21] CentaurSoft Webpage: www.centaursoft.com, retrieved 01.08.2016 

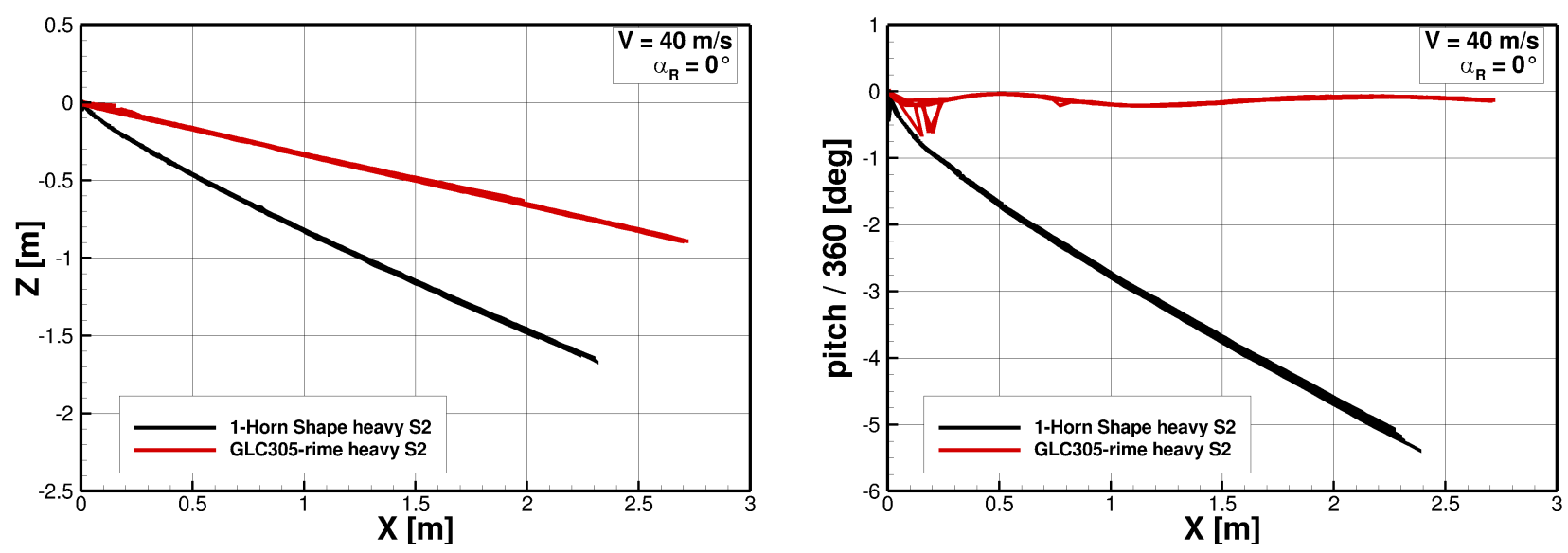

Fig. A.1: Trajectory (left) and pitch angle (right) for a variation of ice block geometry at a freestream velocity of $U_{\infty}=40 \mathrm{~m} / \mathrm{s}$ and a release angle of attack of $\alpha_{R}=0^{\circ}$.
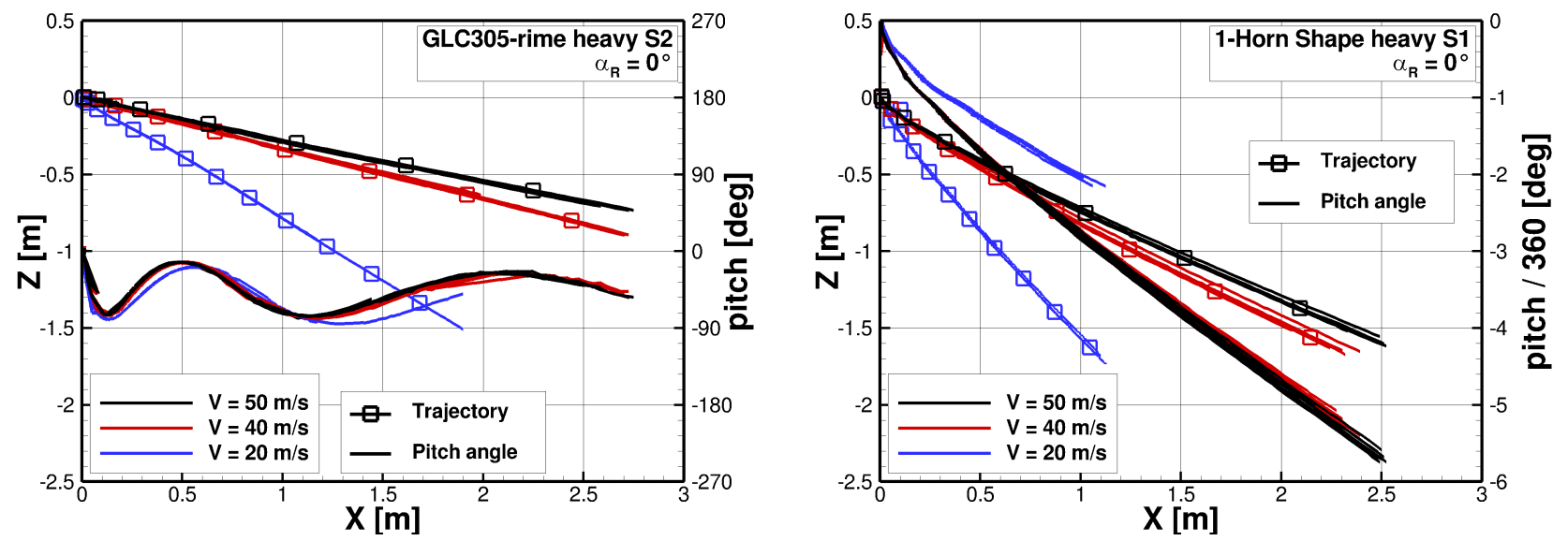

Fig. A.2: Trajectory and pitch angle for a variation of freestream velocity for both ice shapes at a release angle of attack of $\alpha_{R}=0^{\circ}$ (left: GLC305-rime, right: 1-Horn).
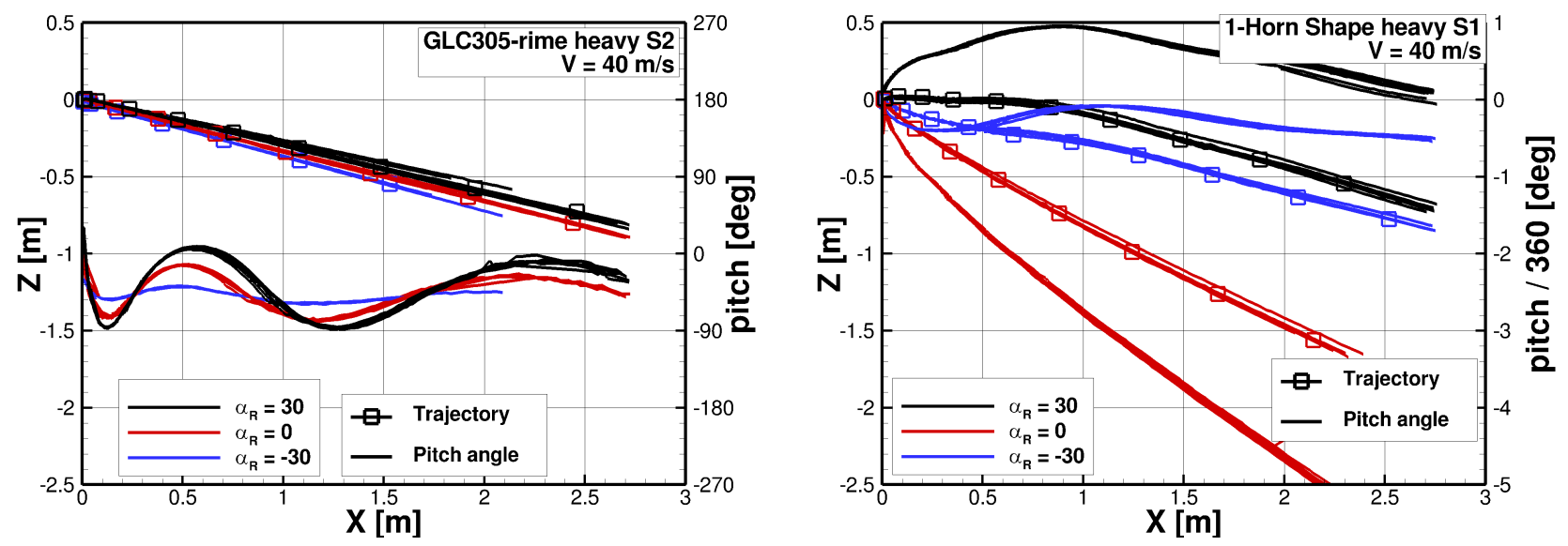

Fig. A.3: Trajectory and pitch angle for a variation of release angle of attack for both ice shapes at a freestream velocity of $U_{\infty}=40 \mathrm{~m} / \mathrm{s}$ (left: GLC305-rime, right: 1 -Horn). 

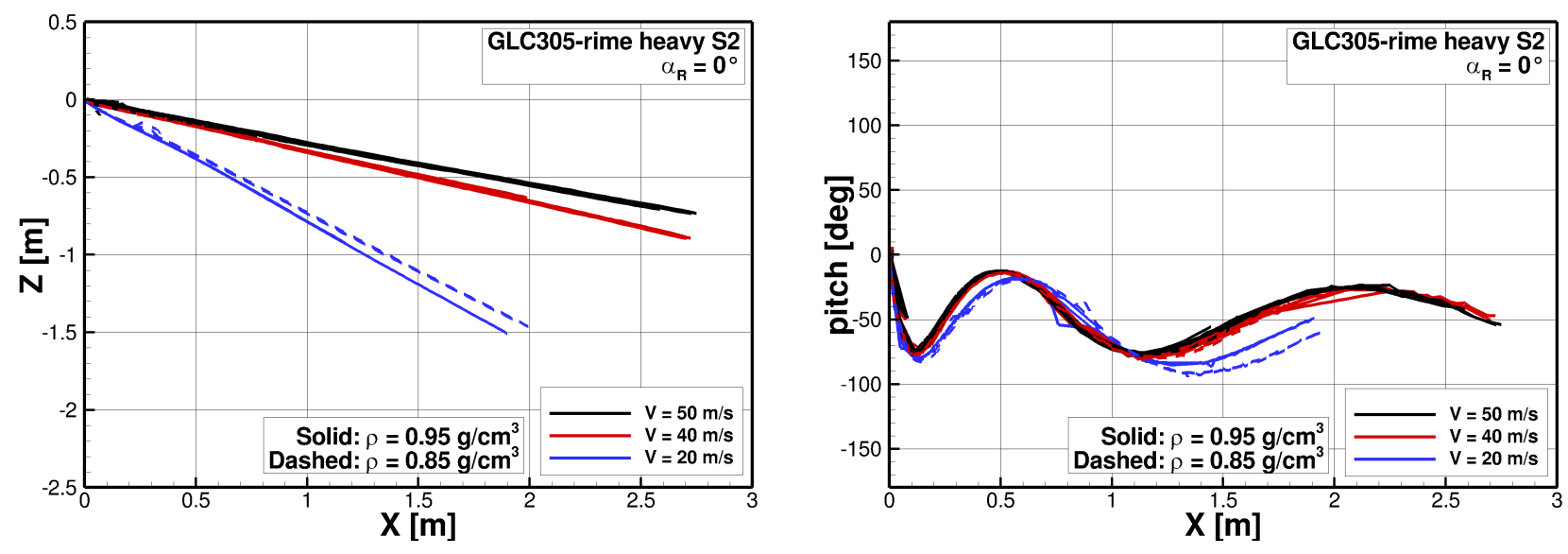

Fig. A.4: Trajectory (left) and pitch angle (right) for a variation of ice block density for the GLC305-rime shape at a release angle of attack of $\alpha_{R}=0^{\circ}$ and various freestream velocities.
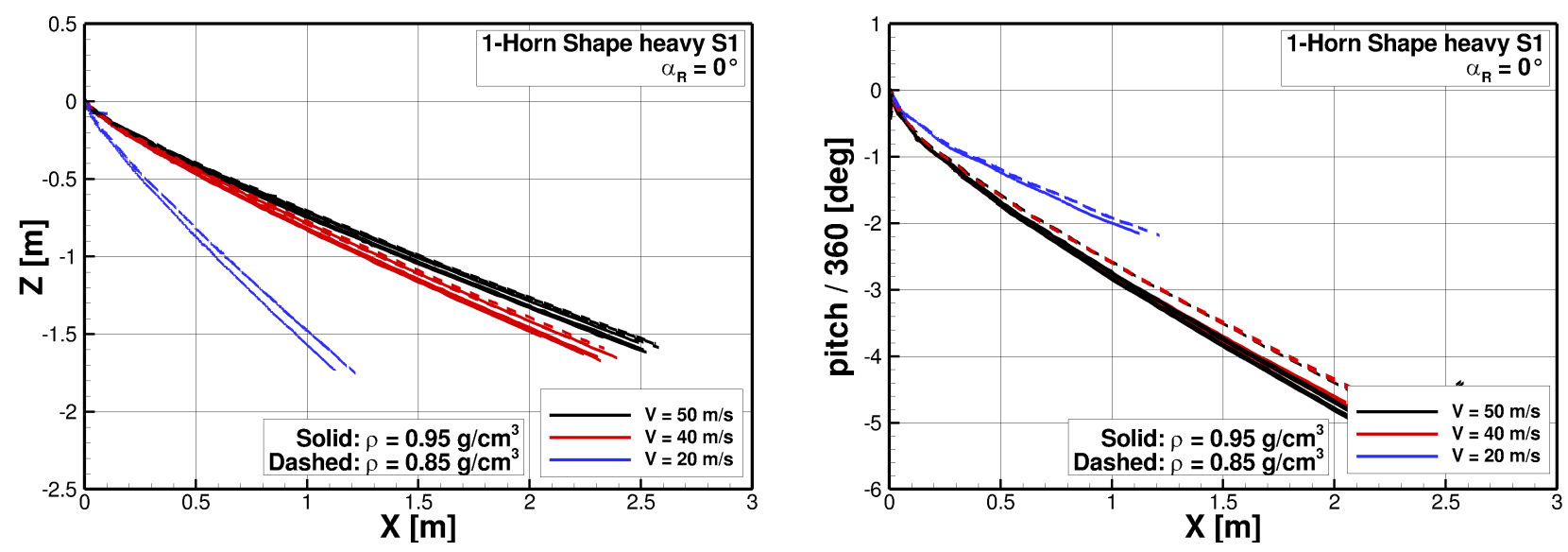

Fig. A.5: Trajectory (left) and pitch angle (right) for a variation of ice block density for the 1-Horn shape at a release angle of attack of $\alpha_{R}=0^{\circ}$ and various freestream velocities.
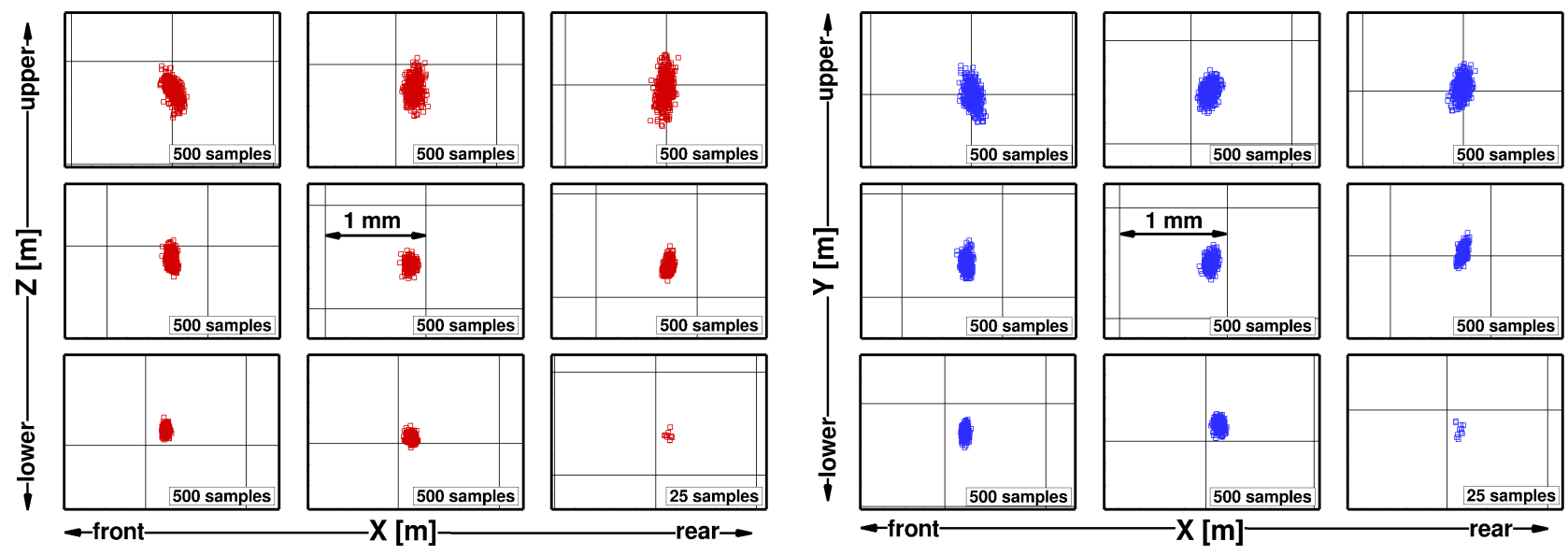

Fig. A.6: Spread of CG position data in the XZ (left) and the XY-plane (right) from accuracy tests. 